Supplementary Materials for

\title{
Regional evapotranspiration from image-based implementation of the Surface Temperature Initiated Closure (STIC1.2) model and its validation across an aridity gradient in the conterminous 5 United States
}

\footnotetext{
Nishan Bhattarai ${ }^{1}$, Kaniska Mallick ${ }^{2}$, Nathaniel A. Brunsell ${ }^{3}$, Ge Sun ${ }^{4}$, Meha Jain ${ }^{1}$

${ }^{1}$ School for Environment and Sustainability, University of Michigan, Ann Arbor, MI 48109, USA

${ }^{2}$ Remote Sensing and Ecohydrological Modeling, Dept. ERIN, Luxembourg Institute of Science and Technology 10 (LIST), L4422, Belvaux, Luxembourg

${ }^{3}$ Geography and Atmospheric Science, University of Kansas, Lawrence, KS 66045, USA

${ }^{4}$ Eastern Forest Environmental Threat Assessment Center, Southern Research Station, U.S. Department of Agriculture Forest Service, Raleigh, 27606, NC, USA

Correspondence to: Nishan Bhattarai (nbhattar@umich.edu)
} 

best overall performance statistics among the three models.

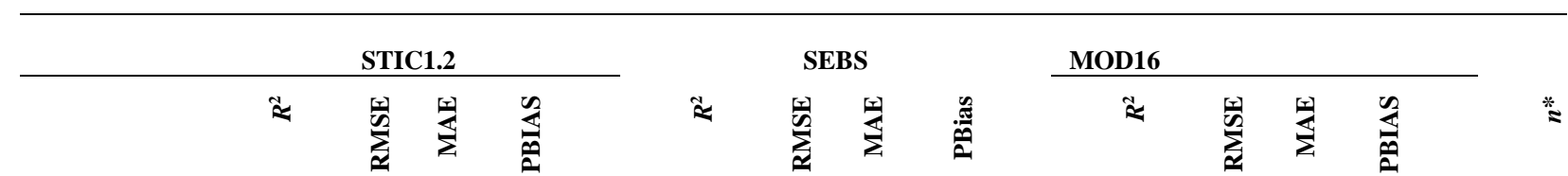

\begin{tabular}{lrrrrrrrrrrrrrr}
\hline US-Me2 & $\mathbf{0 . 6 4}$ & $\mathbf{6 . 6}$ & $\mathbf{5}$ & $\mathbf{- 1 9}$ & 0.64 & 9 & 7 & 33 & 0.43 & 8.1 & 6 & -21 & 80 \\
US-Ton & 0.17 & 9.4 & 7 & 80 & 0.16 & 16.2 & 13 & 179 & $\mathbf{0 . 7 2}$ & $\mathbf{3 . 7}$ & $\mathbf{3}$ & $\mathbf{1 0}$ & 109 \\
US-SRM & $\mathbf{0 . 5}$ & $\mathbf{5 . 8}$ & $\mathbf{4}$ & $\mathbf{2}$ & 0.6 & 7.9 & 7 & 64 & 0.36 & 9.5 & 6 & -71 & 82 \\
US-SRG & $\mathbf{0 . 4 5}$ & $\mathbf{7 . 4}$ & $\mathbf{6}$ & $\mathbf{2}$ & 0.5 & 10 & 8 & 69 & 0.52 & 10.7 & 7 & -68 & 85 \\
US-Wkg & $\mathbf{0 . 3 9}$ & $\mathbf{6 . 3}$ & $\mathbf{5}$ & $\mathbf{1 6}$ & 0.4 & 12.1 & 10 & 119 & 0.53 & 8 & 5 & -64 & 128 \\
US-NR1 & 0.72 & 5.5 & 4 & -14 & $\mathbf{0 . 7 6}$ & $\mathbf{5 . 5}$ & $\mathbf{4}$ & $\mathbf{5}$ & 0.76 & 8.4 & 6 & -44 & 116 \\
US-Kon & 0.82 & 8.6 & 6 & -23 & $\mathbf{0 . 7 2}$ & $\mathbf{8 . 9}$ & $\mathbf{6}$ & $\mathbf{0}$ & 0.87 & 8.2 & 6 & -21 & 59 \\
US-KFS & $\mathbf{0 . 8 1}$ & $\mathbf{7 . 1}$ & $\mathbf{5}$ & $\mathbf{- 1 3}$ & 0.76 & 7.5 & 5 & 2 & 0.77 & 8.5 & 6 & -20 & 88 \\
US-ARM & $\mathbf{0 . 3 8}$ & $\mathbf{1 0}$ & $\mathbf{7}$ & $\mathbf{1 1}$ & 0.45 & 12.2 & 9 & 48 & 0.43 & 10 & 7 & -41 & 61 \\
US-Ne1 & 0.79 & 10.1 & 7 & -32 & $\mathbf{0 . 8 2}$ & $\mathbf{7 . 6}$ & $\mathbf{6}$ & $\mathbf{- 1 5}$ & 0.61 & 16 & 11 & -50 & 114 \\
US-MMS & $\mathbf{0 . 8 9}$ & $\mathbf{5 . 2}$ & $\mathbf{4}$ & $\mathbf{1 1}$ & 0.7 & 8.3 & 6 & 17 & 0.89 & 5.6 & 5 & 12 & 108 \\
US-NC1 & 0.75 & 7.4 & 6 & 16 & 0.65 & 8.9 & 6 & 23 & $\mathbf{0 . 7 7}$ & $\mathbf{6}$ & $\mathbf{4}$ & $\mathbf{7}$ & 75 \\
US-NC2 & 0.88 & 6.1 & 5 & -17 & 0.78 & 7.8 & 6 & -20 & $\mathbf{0 . 8 6}$ & $\mathbf{5}$ & $\mathbf{4}$ & $\mathbf{- 6}$ & 65 \\
\hline
\end{tabular}


a)

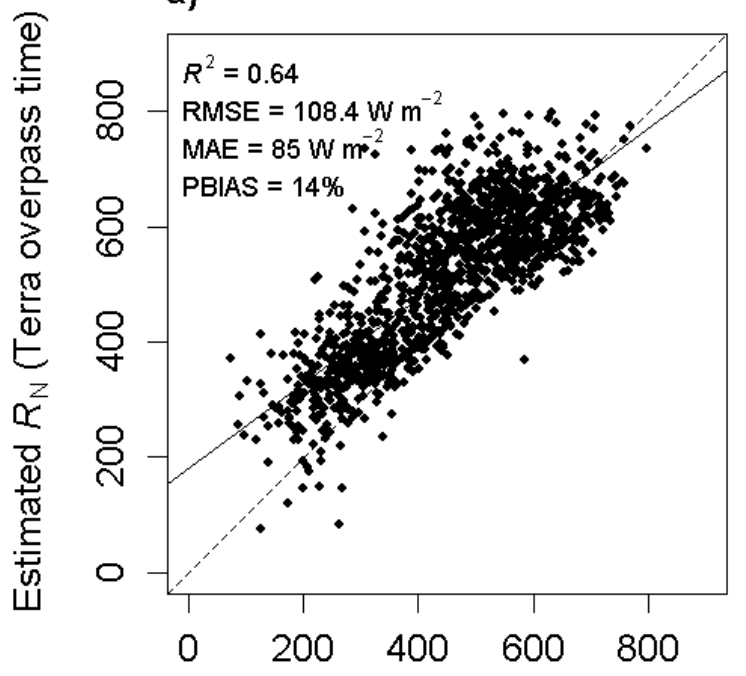

Observed $R_{N}$ (Terra overpass time) b)

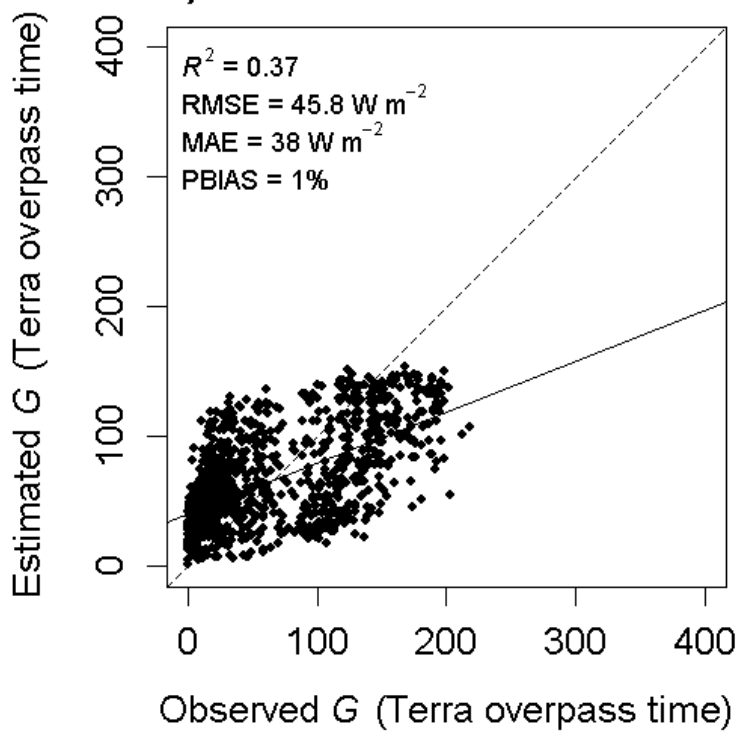

c)

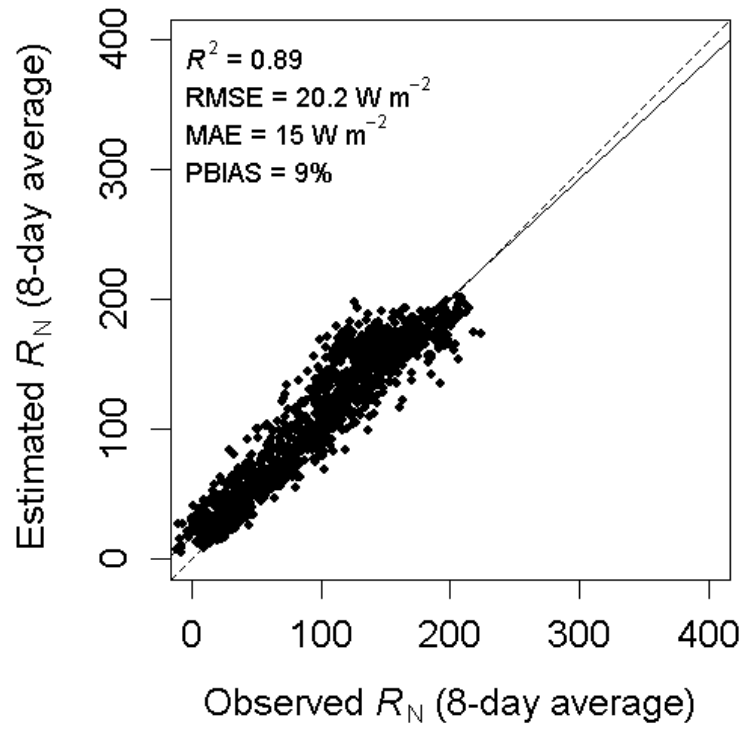

Figure S1: Scatterplots of estimated vs observed (a) $R_{\mathrm{N}}$, (b) $G$, (c) 8-day average daily $R_{\mathrm{N}}$ at the 13 core AmeriFlux sites considered in this study. 

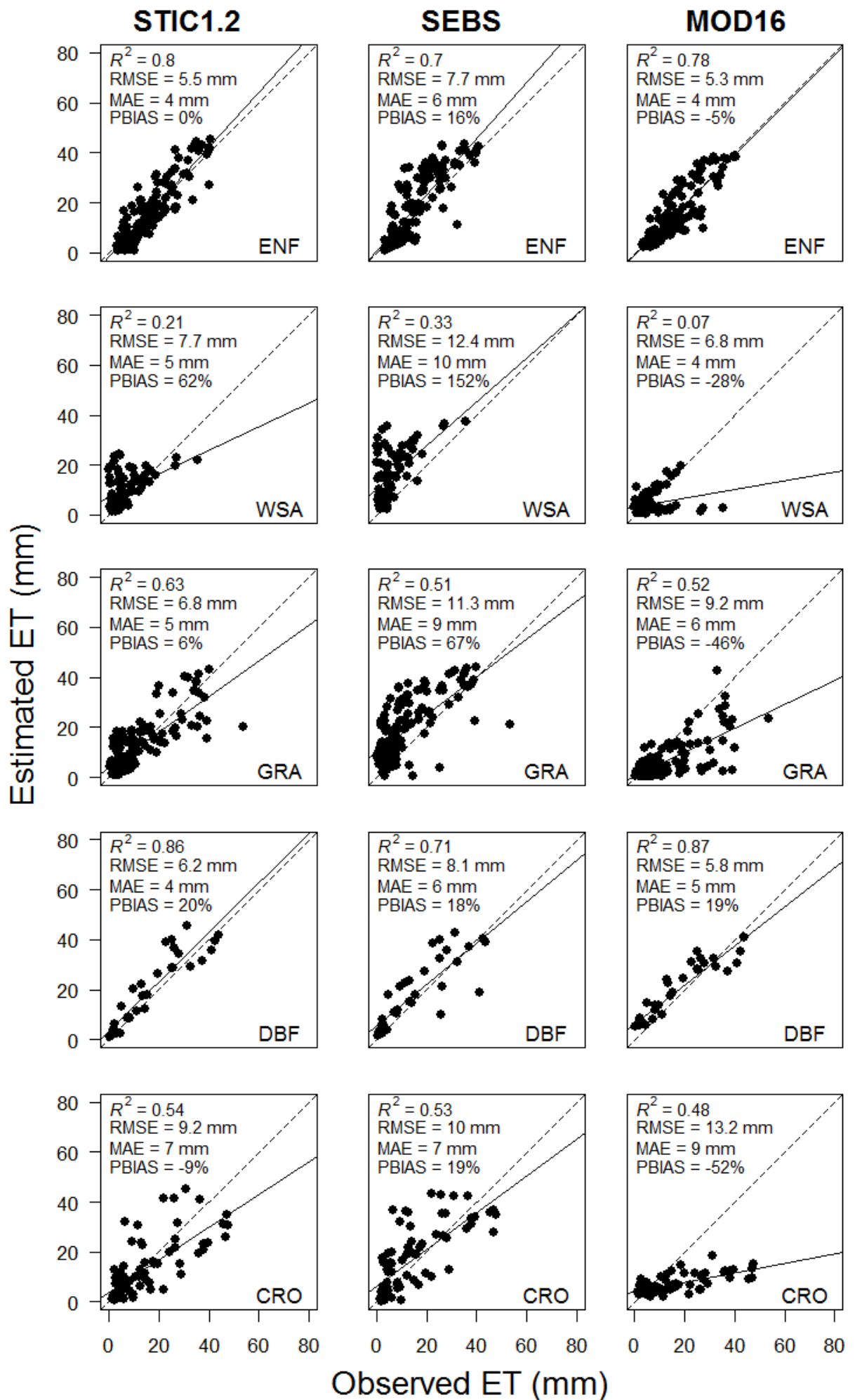

Figure S2: Evaluation of 8-day cumulative ET from STIC1.2, SEBS, and MOD16 aggregated on different biome types against ET observation at the 13 core AmeriFlux sites during dry years. 


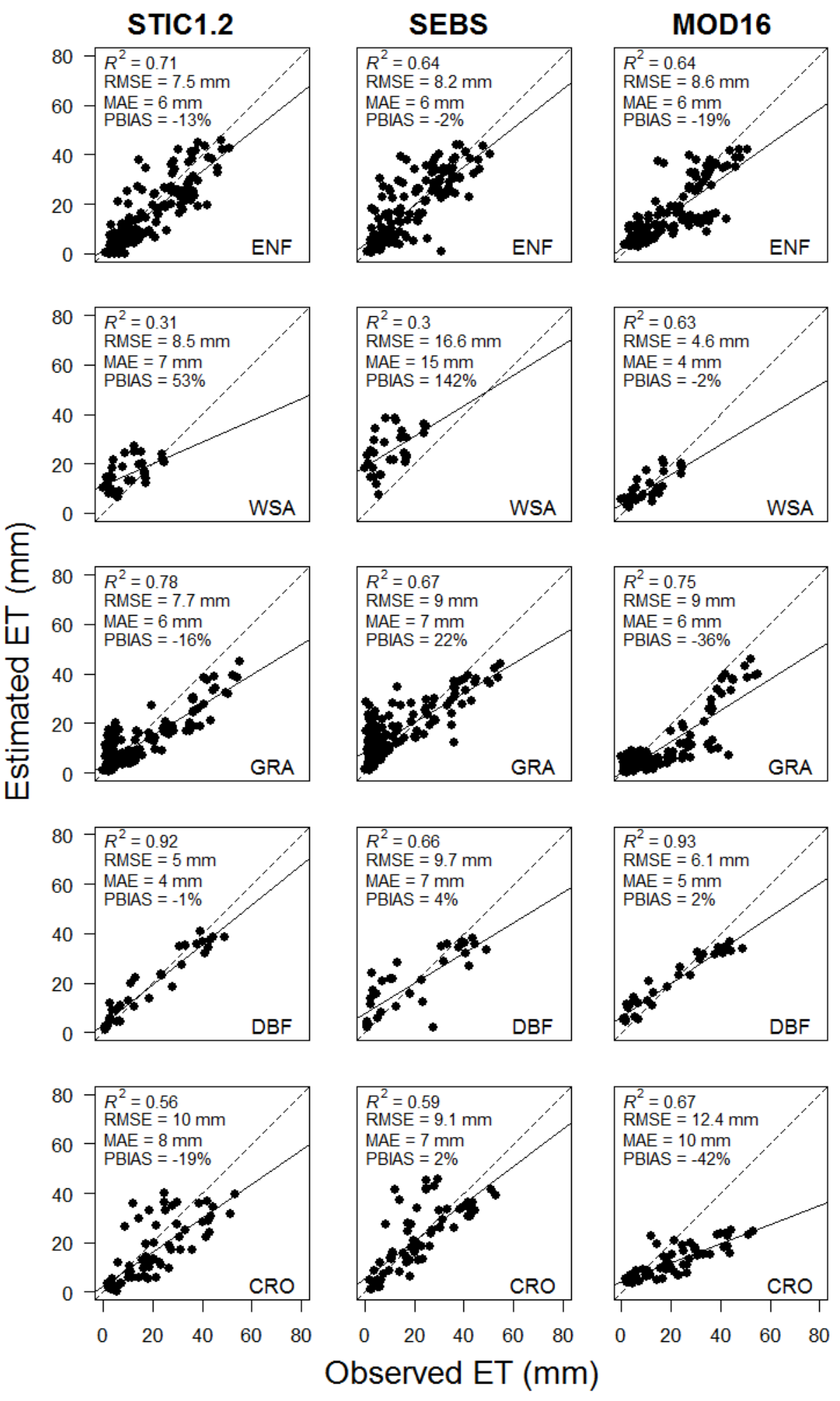

Figure S3: Evaluation of 8-day cumulative ET from STIC1.2, SEBS, and MOD16 aggregated on different biome types against ET observation at the 13 core AmeriFlux sites during wet years. 
STIC1.2
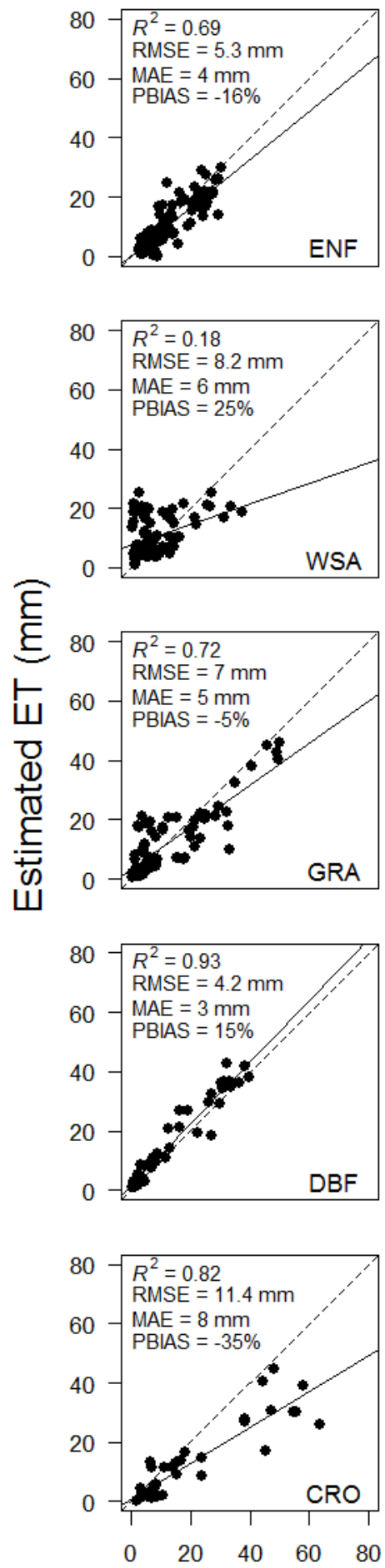
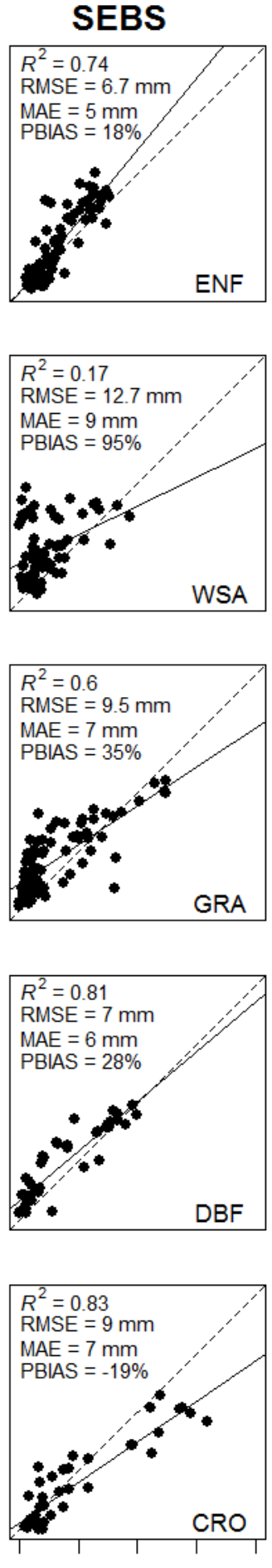

Observed ET (mm)
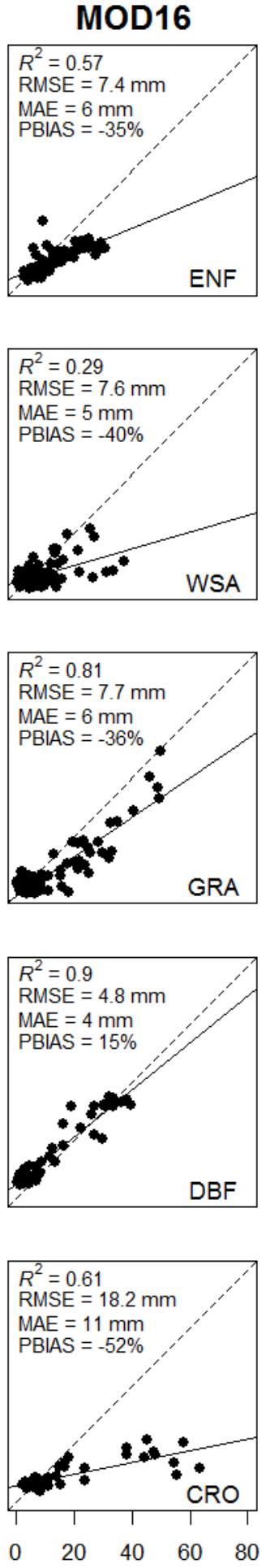

\section{)}

Figure S4: Evaluation of 8-day cumulative ET from STIC1.2, SEBS, and MOD16 aggregated on different biome types against ET observation in the thirteen AmeriFlux sites during normal precipitation year. 

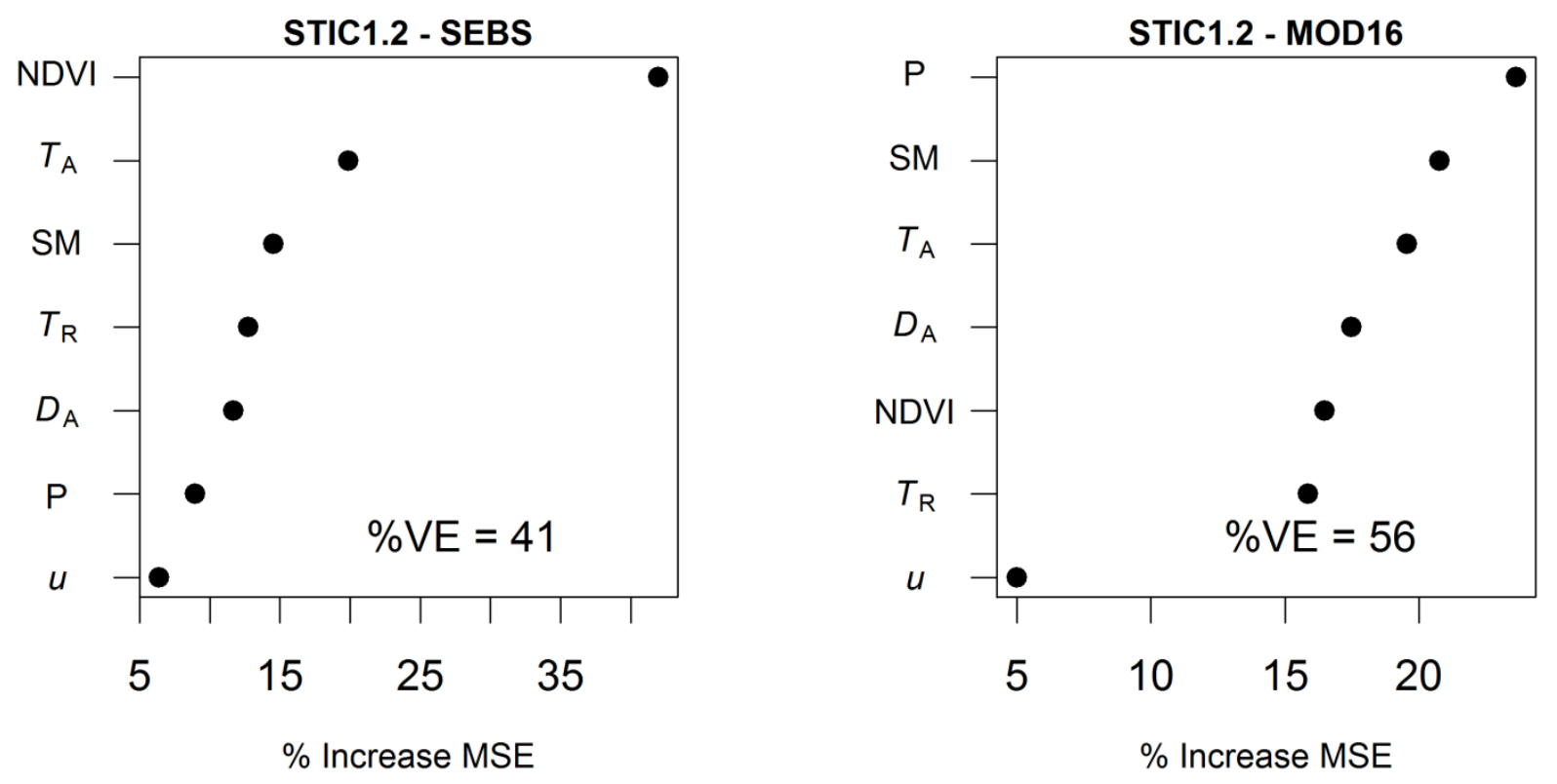

Figure S5: Random forest results for residual difference between 8-day cumulative ET from STIC1.2 and either SEBS or MOD16, when all sites were lumped together. Each plot shows the relative importance of one of seven variables considered in the model, with the most important variable having the largest $\%$ increase in mean squared error (MSE) between the full models and when that variable is permuted. \%VE indicates \% in available variance explained across all trees in the out of bag sample recorded for the full model. 

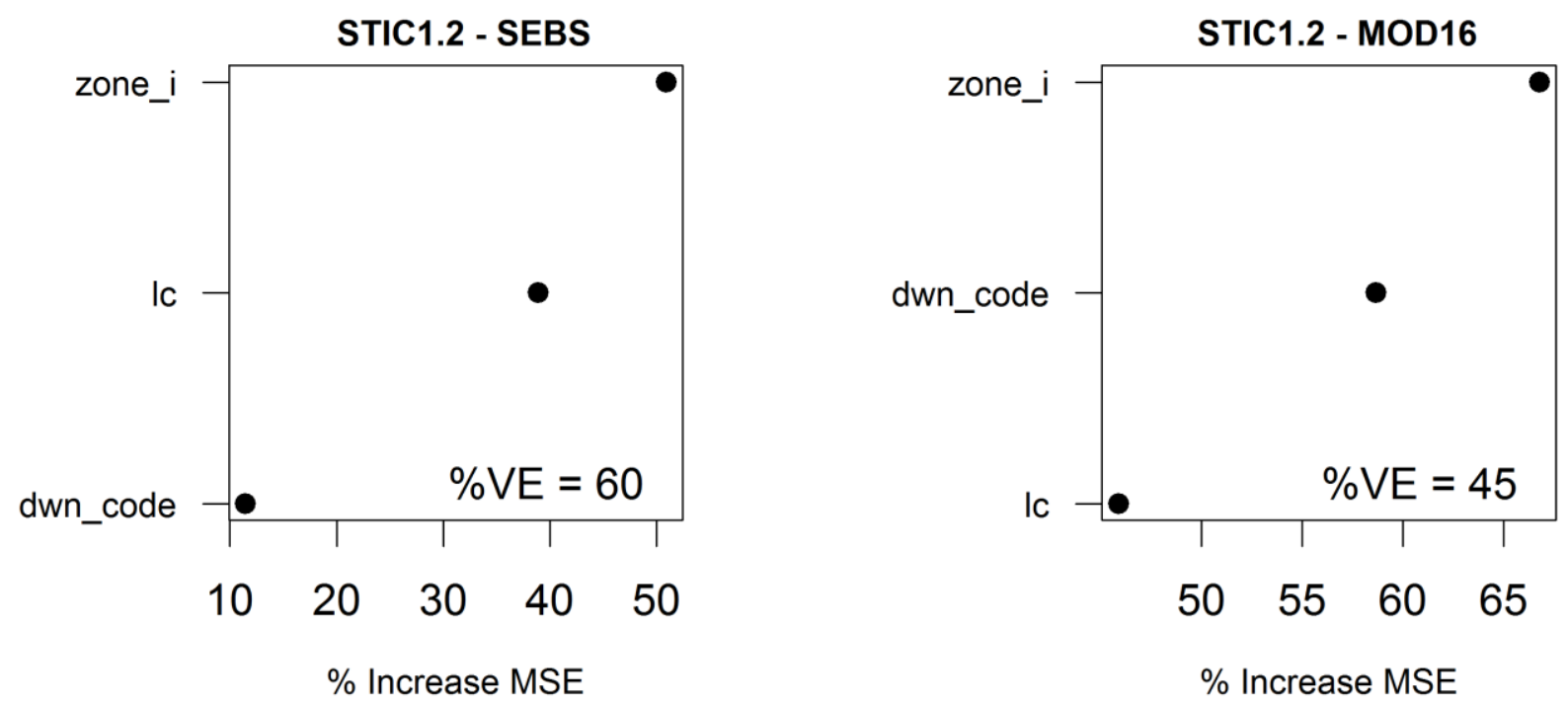

Figure S6: Random forest results for residual difference between annual ET from STIC1.2 and either SEBS or MOD16, when all sites were lumped together. Each plot shows the relative importance of one of three categorical variables considered in the model, with the most important variable having the largest \% increase in mean squared error (MSE) between the full models and when that particular variable is permuted. \% VE indicates $\%$ in available variance explained across all tree in the out of bag sample recorded for the full model. The three categorical variables are for drought conditions [dwn_code $=1($ dry), 2(wet), and 3(normal)], land cover type [lc_year $=$ land cover class], and US zones considered in this study [zone_ $i=$ one of the four zones (W, MW1, MW2, E)]. 